\title{
CRISPR/Cas technology: a revolutionary approach for genome engineering
}

\author{
ZHANG LinLin ${ }^{1,2} \&$ ZHOU Qi ${ }^{1 *}$ \\ ${ }^{1}$ State Key Laboratory of Reproductive Biology, Institute of Zoology, Chinese Academy of Sciences, Beijing 100101, China; \\ ${ }^{2}$ University of Chinese Academy of Sciences, Beijing 100049, China
}

Received April 23, 2014; accepted April 25, 2014; published online May 20, 2014

Citation: Zhang LL, Zhou Q. CRISPR/Cas technology: a revolutionary approach for genome engineering. Sci China Life Sci, 2014, 57: 639-640, doi: $10.1007 / \mathrm{s} 11427-014-4670-\mathrm{x}$

Precision genome editing tools are invaluable for biology, medicine and agriculture. With these tools we can determine the genetic basis underlying biological traits and processes, and then modify the genome of crops and livestock, model organisms, and even of patients, for phenotypic improvement, disease modeling and treatment. Site-specific nucleases provide a powerful and effective approach for precise genome editing in organisms [1]. Two types of site-specific nucleases, namely zinc finger nucleases (ZFNs) and transcription activator-like effector nucleases (TALENs), have already been widely used for genome engineering. Both of them are composed of two functional modules: the DNA binding domain that is programmable for specific DNA sequence binding, and the Fok1 nuclease domain that can induce double strand breaks. By nonhomologous end joining repair, the double strand breaks at target sites can therefore produce sequence insertions and deletions (indels). Recently, a new type of site-specific nucleases, the CRISPR (clustered regularly interspaced short palindromic repeats)/Cas (CRISPR- associated) system, has emerged and raised a revolution in the genomics field.

The CRISPR/Cas system is an acquired immune system of many bacteria and archaea, which can identify the alien plasmids or phages and cut them by the Cas protein. Thus by inserting a plasmid containing Cas genes and specifically designed CRISPRs, an organism's genome can be cut at any desired location. Following this idea, the CRISPR/Cas sys-

*Corresponding author (email: qzhou@ioz.ac.cn) tem was first used for genome editing via specific gene silencing in 2012. In 2013, this system was first adapted for genome editing in mammalian cells, which laid the basis for the widely application of this technology [2,3]. In contrast to ZFN and TALEN, gene targeting of the CRISPR/Cas system is mediated by a small RNA, which make it very easy to design and achieve multiplex genome editing. Till now, the CRISPR/Cas system-mediated gene targeting has been successfully applied in many kinds of species, including plants, flies, zebrafish and rodents [4]. Notably, multiple gene knockout mice and rats, which may need more than two years to construct by conventional embryonic stem cell-based method, could be simultaneously generated in nearly one month in one-step by zygote injection of Cas9 RNA and guide RNAs. By the zygote-injection approach, the CRISPR/Cas system has also produced biallelic knockout pigs, demonstrating the feasibility of this technique in large animals [5]. Recently, gene targeting in non-human primates has also been achieved for the first time through the CRISPR/Cas system [6]. These findings revolutionize the generation of gene-targeting models, which now could be achieved in any species with the help of the CRISPR/Cas system.

Besides gene mutations, the CRISPR/Cas system also has other promising applications in genome manipulation [4]. The RNA-guided properties enable the CRISPR/Cas system to produce genome-wide genetic mutations rather than to modulate individual genes. Several studies using lentivirus-based guide RNA libraries have demonstrated the 
feasibility to use the CRISPR/Cas system for highthroughput genetic screening in human cells. The CRISPR/ Cas system modulate the genome through forming a threecomponent-complex: the Cas9 protein, the guide RNA and the double strand DNA. So the application could be repurposed by modulating the Cas9 function without altering its three-component-complex forming ability. By mutating the nuclease function and fusing the transcription activation or repression domains to Cas9 protein, several groups have successfully activated or silenced endogenous gene expression with the CRISPR/Cas system, suggesting the application potential in gene expression modulation. Another promising potential application of the CRISPR/Cas system is to modify the epigenome. Genome editing technologies like gene knockout can provide direct links between genotypes and phenotypes, and are widely used and play important roles in genetics and functional genomics studies. Thus it is interesting to develop targeted epigenetic editing tools similar to gene knock-out or knock-in. By fusing the endonuclease-mutant Cas9 with an epigenetic modulation domain, such as DNA methylation domain, the CRISPR/ Cas system would be repurposed to modify the epigenetic modifications of targeted regions. It is foreseeable that such epigenetic editing tools will provide a new approach for epigenetics research. Altogether, the CRISPR/Cas system hold great potential to be repurposed to implement more functions including but not limited to above mentioned ones.

However, a major concern of the CRISPR/Cas system has been raised on the potential off-target effects. Several studies revealed that sequence mismatches distal from the protospacer adjacent motif (PAM) at the 5' end of guide RNAs could be tolerated therefore to induce off-target mutagenesis even with frequencies comparable to those observed at the intended on-target sites [7]. In order to reduce or even eliminate the off-target effects, different attempts have been made. One approach is to use a mutant form of Cas 9 protein, the Cas9 nickase, rather than to use Cas 9 endonuclease [8]. These Cas9 mutants simply introduce the offset gap instead of the DNA double strand break, therefore could reduce off-target effects. Studies also showed that the guide RNAs containing 17 or 18 nucleotides have the similar target binding ability (or even higher) compared with the full length guide RNAs, yet their off-target effects were greatly reduced, suggesting truncated guide RNAs may be adopted to avoid off-target effects. The off-target effects would be further reduced by combining the truncated guide RNAs with the Cas9 nickase [8].

In summary, the CRISPR/Cas system provides a new important tool for genome engineering. The easy programmability and high efficiency make it being successfully used in many kinds of species, ranging from plants, flies, fish, rodents, farm animals, to monkeys and human. Besides genome editing, the CRISPR/Cas system can also be easily repurposed to implement versatile functions for many other biological purpose. By further development and improvement of the system, we believe that the CRISPR/Cas system will have important applications in agriculture, biology and medicine.

1 Gaj T, Gersbach CA, Barbas CF 3rd. ZFN, TALEN, and CRISPR/Cas-based methods for genome engineering. Trends Biotechnol, 2013, 31: 397-405

2 Cong L, Ran FA, Cox D, Lin S, Barretto R, Habib N, Hsu PD, Wu X, Jiang W, Marraffini LA, Zhang F. Multiplex genome engineering using CRISPR/Cas systems. Science, 2013, 339: 819-823

3 Mali P, Yang L, Esvelt KM, Aach J, Guell M, DiCarlo JE, Norville JE, Church GM. RNA-guided human genome engineering via Cas9. Science, 2013, 339: 823-826

4 Mali P, Esvelt KM, Church GM. Cas9 as a versatile tool for engineering biology. Nat Methods, 2013, 10: 957-963

5 Hai T, Teng F, Guo R, Li W, Zhou Q. One-step generation of knockout pigs by zygote injection of CRISPR/Cas system. Cell Res, 2014, 24: 372-375

6 Niu Y, Shen B, Cui Y, Chen Y, Wang J, Wang L, Kang Y, Zhao X, Si W, Li W, Xiang AP, Zhou J, Guo X, Bi Y, Si C, Hu B, Dong G, Wang H, Zhou Z, Li T, Tan T, Pu X, Wang F, Ji S, Zhou Q, Huang X, Ji W, Sha J. Generation of gene-modified cynomolgus monkey via Cas9/RNA-mediated gene targeting in one-cell embryos. Cell, 2014, 156: 836-843

7 Pattanayak V, Lin S, Guilinger JP, Ma E, Doudna JA, Liu DR. High-throughput profiling of off-target DNA cleavage reveals RNA-programmed Cas9 nuclease specificity. Nat Biotechnol, 2013, 31: 839-843

8 Ran FA, Hsu PD, Lin CY, Gootenberg JS, Konermann S, Trevino AE, Scott DA, Inoue A, Matoba S, Zhang Y, Zhang F. Double nicking by RNA-guided CRISPR Cas9 for enhanced genome editing specificity. Cell, 2013, 154: 1380-1389

Open Access This article is distributed under the terms of the Creative Commons Attribution License which permits any use, distribution, and reproduction in any medium, provided the original author(s) and source are credited. 